\author{
Mandy M. A. Conijn \\ Jeroen Hendrikse \\ Jaco J. M. Zwanenburg \\ Taro Takahara \\ Mirjam I. Geerlings \\ Willem P. Th. M. Mali \\ Peter R. Luijten
}

\section{Perforating arteries originating from the posterior communicating artery: a 7.0-Tesla MRI study}

Received: 10 February 2009

Revised: 5 May 2009

Accepted: 14 May 2009

Published online: 17 June 2009

(C) The Author(s) 2009.

This article is published with open access at Springerlink.com

M. M. A. Conijn $(\bowtie) \cdot$ J. Hendrikse · T. Takahara - W. P. T. M. Mali •

\section{P. R. Luijten}

Department of Radiology

(Hp E 01.132),

University Medical Center Utrecht,

P.O. Box 85500, 3508 GA

Utrecht, The Netherlands

e-mail: M.Conijn@umcutrecht.nl

Tel.: +31-88-7551234

Fax: +31-30-2581098

M. M. A. Conijn - M. I. Geerlings Julius Center for Health Sciences and Primary Care,

University Medical Center Utrecht, Utrecht, The Netherlands

J. J. M. Zwanenburg · P. R. Luijten Image Sciences Institute,

University Medical Center Utrecht,

Utrecht, The Netherlands
Abstract The aim of this study was to investigate the ability of time-of-flight (TOF) magnetic resonance (MR) angiography at 7.0 Tesla to show the perforating branches of the posterior communicating artery ( $\mathrm{PCoA})$, and to investigate the presence of such visible perforating branches in relation to the size of the feeding PCoA. The secondary aim was to visualise and describe the anterior choroidal artery and the perforating branches of the P1segment of posterior cerebral artery (P1). Forty-six healthy volunteers underwent TOF MR angiography at 7.0 Tesla. With 7.0-Tesla imaging, we visualised for the first time perforating arteries originating from the $\mathrm{PCoA}$ in vivo without the use of contrast agents. A perforating artery from the PCoA was found in a large proportion of the PCoAs (64\%). The presence was associated with a larger diameter of the underlying PCoA (1.23 versus $1.06 \mathrm{~mm}, P=0.03$ ). The anterior choroidal artery was visible bilaterally in all participants. In $83 \%$ of all $\mathrm{P} 1 \mathrm{~s}$, one or two perforating branches were visible. Non-invasive assessment of the perforating arteries of the PCoA together with the anterior choroidal artery and the perforating arteries of the P1 may increase our understanding of infarcts in the deep brain structures supplied by these arteries.

Keywords Magnetic resonance angiography · Circle of Willis . Posterior communicating artery . Perforating arteries.

Lacunar infarction
Abbreviations MR: Magnetic resonance $\cdot$ PCoA: Posterior communicating artery $\cdot \mathrm{P} 1$ : P1- segment of posterior cerebral artery . iaDSA: Intra-arterial digital subtraction angiography TOF: Time of flight - SAR: Specific absorption rate $\cdot$ FOV: Field of view - TONE: Tilt optimised non-saturated excitation . MIP: Maximum intensity projection . TR: Repetition time - TE: Echo time . SENSE: Sensitivity encoding

\section{Introduction}

The posterior communicating artery $(\mathrm{PCoA})$ is an important component of the circle of Willis. A large variability exists in the configuration of the PCoA, which can be large, hypoplastic or even absent in some cases. In addition, two configurations of the posterior part of the circle of Willis can be distinguished: a non-foetal configuration, when the diameter of the PCoA is smaller than the diameter of the ipsilateral P1 segment of the posterior cerebral artery (P1), and a foetal configuration, when the diameter of the PCoA is larger than the diameter of the ipsilateral P1.

A small or absent PCoA appears to be a risk factor for ischaemic lesions of the brain. Ischaemic cerebral infarctions are more often found in patients with a small or absent PCoA than in patients with a large PCoA, independent of the presence of internal carotid artery occlusion [1,2]. A foetal variant of the PCoA has been found to be less common in patients with occipital lobe infarctions than in healthy controls [3]. Besides ischaemic cerebral infarctions, the 
PCoA also seems to be an important determinant in the aetiology of white-matter lesions. A study among patients with clinical manifestations of atherosclerotic disease demonstrated a decreased deep white-matter lesion load in patients with a foetal configuration of the circle of Willis [4], although these results were not confirmed in a different population [5].

Lacunar infarctions are thought to result from occlusion of deep perforating arteries, also called the thalamoperforating arteries [6, 7]. A potential protective effect of a large PCoA against ischaemic brain lesions may be explained by the perforating arteries branching from the PCoA, which feed the deep brain structures and deep white matter [1-4]. Thus far, these perforating arteries could only be assessed post-mortem or via intra-arterial digital subtraction angiography (iaDSA) [8, 9] or rotational angiography [10]. Therefore, few studies have investigated the perforating arteries, and little is known about the perforating arteries branching from the PCoA. Recently, MR angiography at the field strength of 7.0 Tesla was introduced as a non-invasive alternative to depict the perforating branches of the circle of Willis [11]. This ultra-high field strength makes it possible to visualise submillimeter vessels for the first time in vivo without the use of any contrast agents.

The primary aim of the present study is to assess the ability of time-of-flight MR angiography at 7.0 Tesla to show the perforating branches of the posterior communicating artery, and to investigate the presence of such perforating branches in relation to the size of the feeding posterior communicating artery and the configuration of the posterior part of the circle of Willis. The secondary aim is to assess the ability to visualise the perforating branches of the $\mathrm{P} 1$, and to describe these branches and to describe the anterior choroidal artery.

\section{Materials and methods}

\section{Participants}

Forty-six healthy volunteers [ 25 men and 21 women, mean age of 30 (SD 12.6) years] from the local university were included. None of them reported a history of neurological or vascular disease.

The study was approved by the medical ethics committee of the Medical Center in Utrecht, and written informed consent was obtained from all participants.

\section{MR imaging}

MR imaging was performed with a 7.0-Tesla whole-body system (Philips Healthcare, Cleveland, OH, USA), using a volume transmit and a 16-channel receive-only head coil (Nova Medical, Wilmington, MA, USA). Time-of-flight (TOF) MR angiography was performed in each participant using a turbo field echo sequence, with a presaturation slab positioned superior to the imaging volume to suppress the venous blood and applied once per $230 \mathrm{~ms}$ to avoid specific absorption rate (SAR) constraints. Two variants of the TOF angiography were used, with a slightly different resolution. In the first variant, the acquired voxel size was $0.6 \times 0.6 \times 0.6 \mathrm{~mm}^{3}$, for the second variant it was $0.4 \times 0.5 \times$ $1.0 \mathrm{~mm}^{3}$. The other imaging parameters were as follows: field of view (FOV) $200 \times 181 \times 68 \mathrm{~mm}$; acquired matrix of $332 \times 294$ for the variant with $0.6 \times 0.6 \mathrm{~mm}$ in-plane resolution and $500 \times 354$ for the variant with $0.4 \times 0.5 \mathrm{~mm}$ in-plane resolution; repetition time (TR) $23 \mathrm{~ms}$ and echo time (TE) 2.3-2.6 ms, depending on the resolution and angulation. Excitation pulses consisted of tilt-optimised non-saturated excitation (TONE) pulses with nominal flip angle variation of $16-24^{\circ}$ in the feet-head direction over the slab. The slab was acquired in a series of four thinner chunks (17.5 mm each) to increase the inflow effect. Sensitivity encoding (SENSE) was applied in the RL direction with an acceleration factor of 3 . The images were reconstructed to $0.3-\mathrm{mm}$ isotropic voxels, and the built-in phase correction and partial-echo filter of the scanner were applied during reconstruction. The imaging duration was approximately $9 \min 30 \mathrm{~s}$.

\section{Post-processing}

All images were exported to an offline workstation, equipped with the same viewing software as the 7.0-Tesla MR system. The post-processing of the angiographic data was performed on the standard console. Maximum intensity projections (MIPs) were reconstructed for transverse slabs (thickness $3 \mathrm{~mm}, 2-\mathrm{mm}$ overlap, 30 slices) and for sagittal slabs (thickness $3 \mathrm{~mm}, 2-\mathrm{mm}$ overlap, 50 slices and thickness $0.3 \mathrm{~mm}$, no overlap, 250 slices), and a coronal reconstruction (thickness $0.3 \mathrm{~mm}$, no overlap, 150 slices) was made from the axial source dataset of the TOF MR angiograms.

On the transverse slab MIP, intensity profiles for both the left and right $\mathrm{P} 1$ were obtained, as illustrated in Fig. $1(\mathrm{a}, \mathrm{b})$. The software programme Matlab (v. 7.6, MathWorks, Natick, MA) was used to calculate the full width at half maximum (FWHM) of the profiles. We took the FWHM as an estimate of the diameter of the P1.

The thin $0.3-\mathrm{mm}$ sagittal slab MIP was used to investigate whether the PCoA was visible on the left and the right sides and, when a PCoA was found, to identify perforating arteries branching from the PCoA. If no perforating branches were visible, an intensity profile was made at one point of the PCoA, and the FWHM was calculated to estimate the diameter. If an image did show a perforating artery branching from the PCoA, intensity profiles were obtained both anterior to the branch and posterior to the branch. For the overall diameter of the PCoA, the mean of the diameters of the anterior and posterior of the branch was calculated. To estimate the 

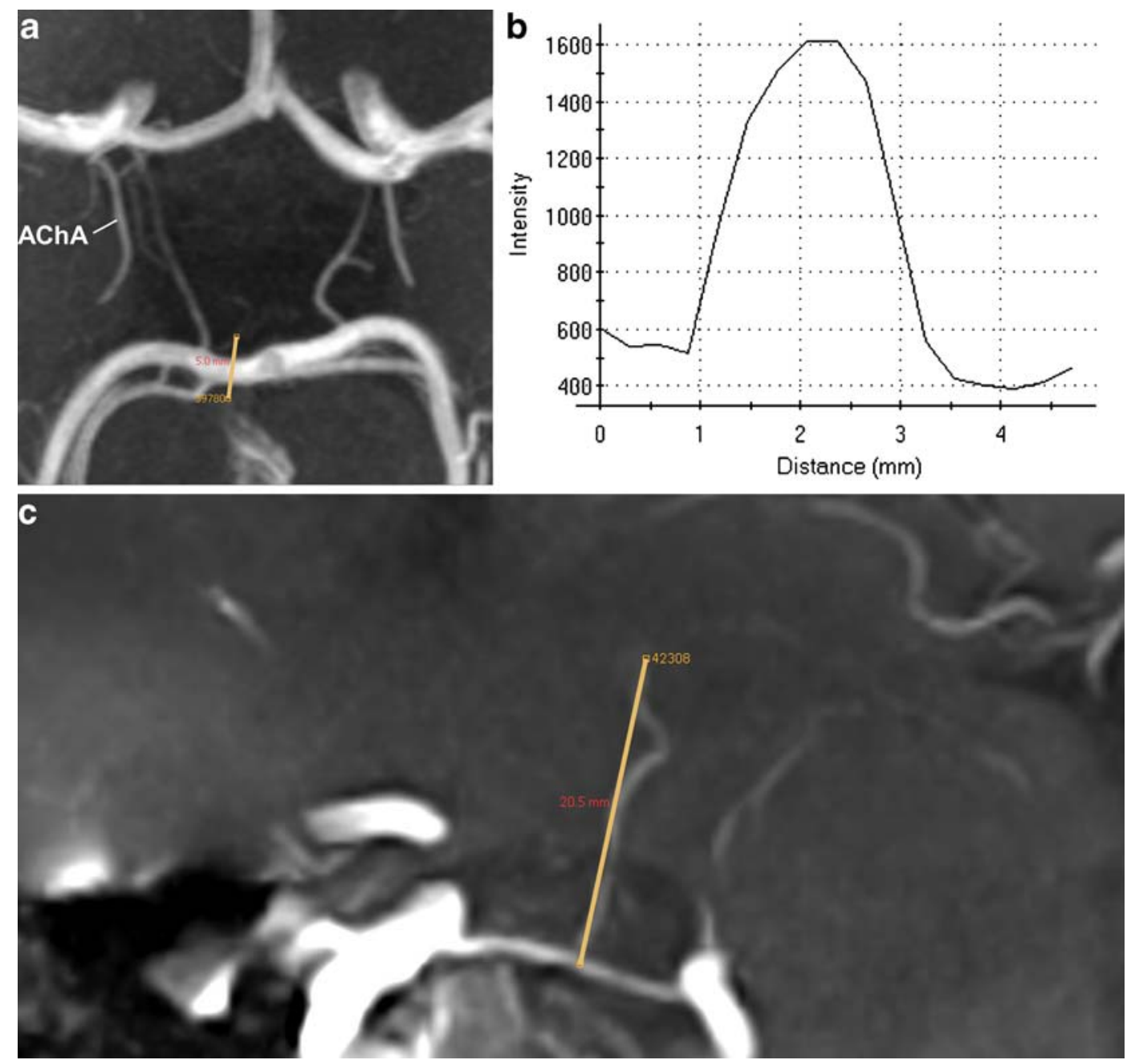

Fig. 1 a Time-of-flight angiography image of the circle of Willis and the anterior choroidal artery (AChA) on 7.0-Tesla MRI, transverse slab maximum intensity projection (thickness $7 \mathrm{~mm}$ ). A line was set perpendicular to the left P1, and the corresponding intensity profile (b) is shown. c The posterior communicating artery

diameter of the branch itself, an additional intensity profile was obtained for which the FWHM was calculated.

To obtain a rough estimate of the length of the perforating branches, measurements were performed by drawing a line from the most distal part, where the branch was still visible, back to the origin of the branch at the level of the PCoA on the sagittal 7-mm slab MIP reconstructions (Fig. 1c). Although this method does not take the curvature of the vessel into account and is measured on a slab MIP projection, this measurement was only used to get an indication of the length over which the perforators are visible on 7.0-Tesla MR angiography.

The 3-mm sagittal slab MIP was used to obtain intensity profiles of the left and right anterior choroidal artery to calculate the FWHM. Because of the variation in diameter of the anterior choroidal artery, three intensity profiles were obtained per artery between the origin of the artery at the with a perforating branch on a sagittal slab maximum intensity projection (thickness $7 \mathrm{~mm}$ ) is shown. A line was set from the origin of the perforator to the last point at which the perforator was visible to obtain a rough estimate of the length of the perforator

internal carotid artery and the point where the anterior choroidal artery diverges from the PCoA. The mean of these three measurements was taken as the diameter of the anterior choroidal artery.

The presence of perforating branches from the P1 was investigated on the thin $0.3-\mathrm{mm}$ coronal reconstruction, and the branches from the left and right P1 were counted. Because of the relatively low contrast between the arteries and the background and their rather tortuous course, it was not possible to obtain a diameter or length measurement of these arteries.

\section{Statistical analysis}

In participants in whom a PCoA was found, we used the unpaired $t$-test to test for differences in PCoA diameter 
between PCoAs with and without a perforating branch. The sides on which no PCoA was found were left out of the analysis.

The two configurations of the posterior part of the circle of Willis, the non-foetal configuration (diameter PCoA < diameter P1) and the foetal configuration (diameter PCoA $>$ diameter P1), were analysed. The presence of a perforating PCoA branch was compared in these two configurations with use of the chi-squared test.

For all measurements together and for the left and the right hemispheres separately, the correlation between the diameter of the P1 and the diameter of the ipsilateral PCoA were evaluated. The correlation between the left and right hemispheres was analysed for the diameter of the P1, the diameter of the PCoA and the diameter of the P1 divided by the diameter of the PCoA ( $1 / / \mathrm{PCoA}$ ratio). A $P$ value $<$ 0.05 was considered significant.

\section{Results}

Figure 2 shows a typical example of a perforating artery branching from the PCoA in a sagittal slab MIP.

There was bilateral absence of a visible PCoA in 2 of the 46 participants $(4.4 \%)$, unilateral absence was found in 7 $(15.2 \%)$, and in 37 participants $(80.4 \%)$ the PCoA was visible bilaterally (Table 1). Taking the left and right hemispheres together, a PCoA was visible in 81 of the 92 hemispheres $(88.1 \%)$, of which $52(64.2 \%)$ had a visible perforating artery and $29(35.8 \%)$ had no perforating artery.

Within the left hemisphere, $39(84.8 \%)$ of the 46 participants had a visible PCoA, of whom $23(59.0 \%)$ showed a perforator and $16(41.0 \%)$ did not. In the right hemisphere, a PCoA was found in 42 out of 46 participants $(91.3 \%), 29(69.0 \%)$ with a perforator and $13(31.0 \%)$ without a perforator. Two visible perforators branching from one PCoA were found for two PCoAs, both in the right hemisphere (Fig. 3).

The mean diameter of the perforating arteries was $0.72 \mathrm{~mm}$ (SD 0.15). With the method we used to indicate

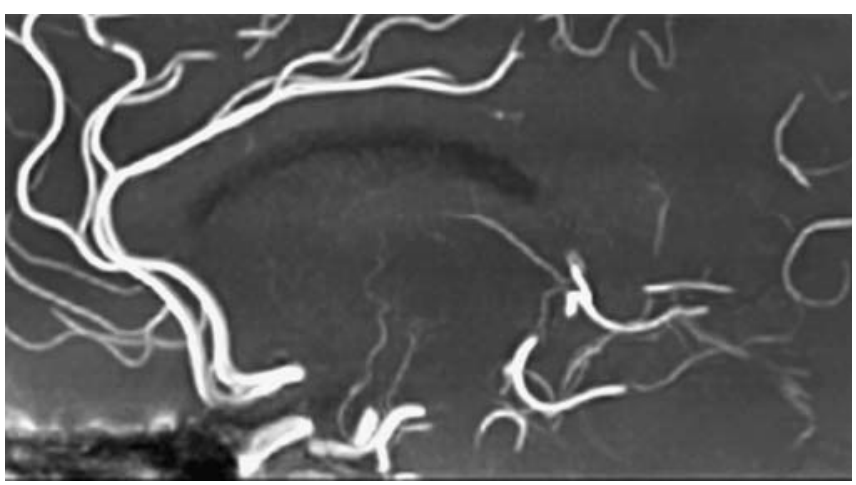

Fig. 2 Time-of-flight angiography image of the posterior communicating artery with a perforating branch on 7.0-Tesla MRI. Sagittal slab maximum intensity projection (thickness $7 \mathrm{~mm}$ )
Table 1 Unilateral and bilateral absence and presence of a visible PCoA

\begin{tabular}{ll}
\hline PCoA & Participants/hemispheres (\%) \\
\hline Bilateral absence & $02 / 04(4.3 \%)$ \\
Unilateral absence & $07 / 14(15.2 \%)$ \\
Bilateral presence & $37 / 74(80.4 \%)$ \\
Total & $46 / 92(100 \%)$ \\
\hline
\end{tabular}

the length, the mean length of the perforating arteries was $19.3 \mathrm{~mm}$ (SD 6.4), ranging from 6.1 to $36.0 \mathrm{~mm}$.

No correlation was found between the diameter of the $\mathrm{P} 1$, the diameter of the PCoA or the presence of a perforating artery and age or gender.

The mean diameter of all PCoAs with a visible accompanying perforator was $1.23 \mathrm{~mm}$ (SD 0.35); this was $1.22 \mathrm{~mm}$ (SD 0.33) for the left hemisphere and $1.24 \mathrm{~mm}$ (SD 0.38) for the right hemisphere. For the PCoAs without a perforator present, the mean diameter was smaller, $1.06 \mathrm{~mm}$ (SD 0.34) for all PCoAs together; $1.05 \mathrm{~mm}$ (SD 0.32) for the left hemisphere and $1.07 \mathrm{~mm}$ (SD 0.38) for the right hemisphere. Taking the left and right hemispheres together, the overall difference in diameter was $0.18 \mathrm{~mm}(\mathrm{SD} 0.08, P=0.030)$ in favour of the PCoA with a perforator; $0.18 \mathrm{~mm}$ (SD $0.11, P=0.101)$ for the left and $0.18 \mathrm{~mm}$ (SD 0.13, $P=0.172)$ for the right hemispheres separately.

Of the 81 hemispheres in which a PCoA was present, there was a foetal configuration (diameter PCoA > diameter P1) in 16 hemispheres and a non-foetal configuration (diameter $\mathrm{PCoA}<$ diameter P1) in 65 hemispheres. Of those with a foetal configuration, $12(75.0 \%)$ had a visible perforating branch; this was $40(61.5 \%)$ for the participants with a non-foetal configuration (Table 2). Although a perforating branch was present more often in cases of foetal configuration, this difference was not significant $(P=0.392)$.

Furthermore, we analysed the correlation between the diameter of the $\mathrm{P} 1$ and the diameter of the PCoA. Overall, a

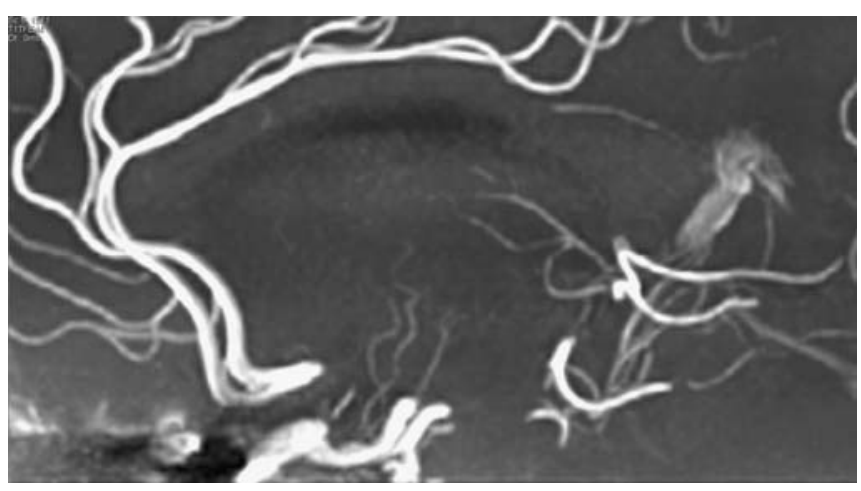

Fig. 3 Time-of-flight angiography image of the posterior communicating artery with two perforating branches. Sagittal slab maximum intensity projection (thickness $10 \mathrm{~mm}$ ) 
Table 2 Configuration of the posterior part of the circle of Willis and presence of a perforator

\begin{tabular}{lll}
\hline Configuration & Hemispheres $(\%)$ & Perforator present/not present $(\%)$ \\
\hline Foetal $(\mathrm{PCoA}>\mathrm{P} 1)$ & $16(17.4 \%)$ & $\begin{array}{l}\text { Present: } 12(75.0 \%) \\
\text { Not present: } 4(25.0 \%)\end{array}$ \\
Non-foetal $(\mathrm{PCoA}<\mathrm{P} 1)$ & $65(70.7 \%)$ & $\begin{array}{l}\text { Present: } 40(61.5 \%) \\
\text { Not present: } 25(38.5 \%)\end{array}$ \\
Subtotal & $81(88.1 \%)$ & $\begin{array}{l}\text { Present } 52(64.2 \%) \\
\text { Not present: } 29(35.8 \%)\end{array}$ \\
No PCoA & $11(11.9 \%)$ & $\begin{array}{l}\text { Present: } 0(0 \%) \\
\text { Not present: } 11(100 \%) \\
\text { Total }\end{array}$ \\
& $92(100 \%)$ & $\begin{array}{l}\text { Present: } 52(56.5 \%) \\
\text { Not present: } 40(43.5 \%)\end{array}$ \\
\end{tabular}

correlation coefficient of $-0.575(P<0.001$, Fig. 4) was found; for the P1 and the PCoA in the left hemisphere separately, the correlation coefficient was $-0.598(P<$ $0.001)$ and for the right hemisphere $-0.663(P<0.001)$.

The data showed a correlation between the left and the right halves of the posterior part of the circle of Willis for the diameter of the P1 $(R=0.457, P=0.002)$, the diameter of the PCoA $(R=0.554, P<0.001)$ and for the $\mathrm{P} 1 / \mathrm{PCoA}$ ratio $(R=0.462, P=0.005)$.

A visible anterior choroidal artery was found bilaterally in all participants, with a mean diameter of $0.83 \mathrm{~mm}$ (SD 0.14).

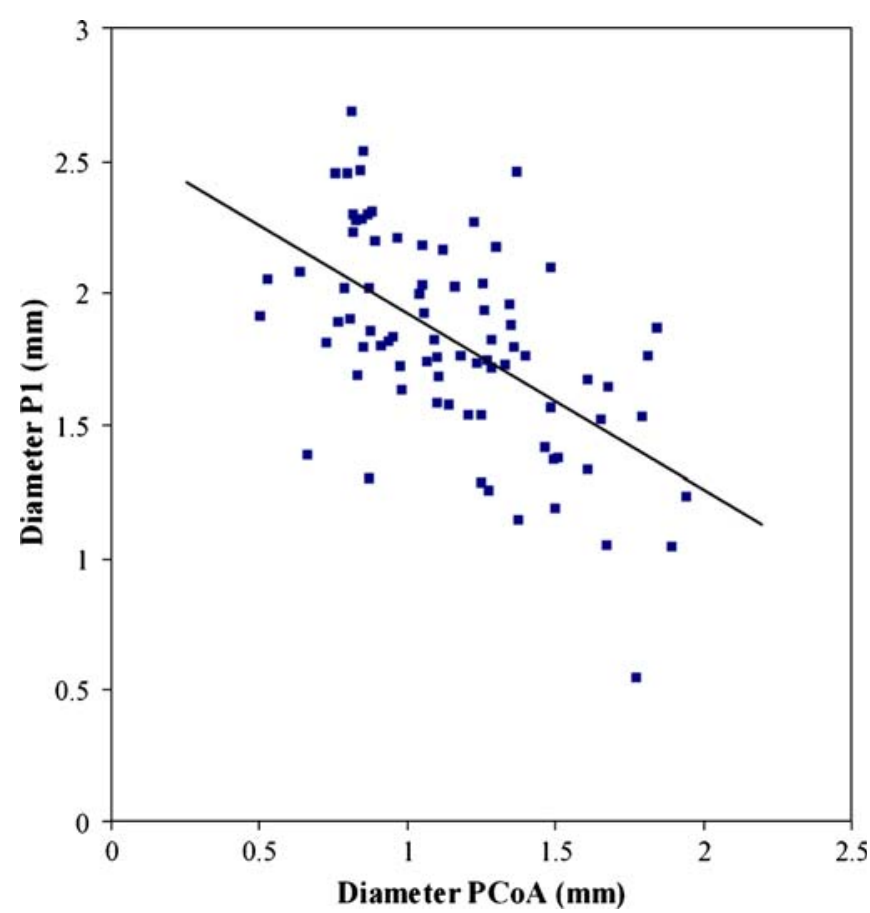

Fig. 4 Correlation between the diameter of the P1 and the PCoA for $\mathrm{P} 1$ and the PCoA of the left and right sides together. Correlation coefficient $-0.575(P<0.001)$. P1 P1 segment of the posterior cerebral artery, $P C O A$ posterior communicating artery
Figure 5 shows an example of perforating arteries originating from the left and right $\mathrm{P} 1$. Taking the left and right $\mathrm{P} 1$ together, no perforators were visible in $16 \%$ of the $\mathrm{P} 1 \mathrm{~s}$, one single perforator was visible in $63 \%$ and two visible perforators were present in $20 \%$ of the P1s. There was no significant difference in diameter between a P1 with one or two perforating arteries and a P1 without a perforating artery.

\section{Discussion}

Time-of-flight MR angiography at 7.0 Tesla showed the presence of a clearly visible perforating branch of the PCoA on 52 of the 81 sides on which a PCoA was present $(64.2 \%)$. The presence of a perforating branch from the

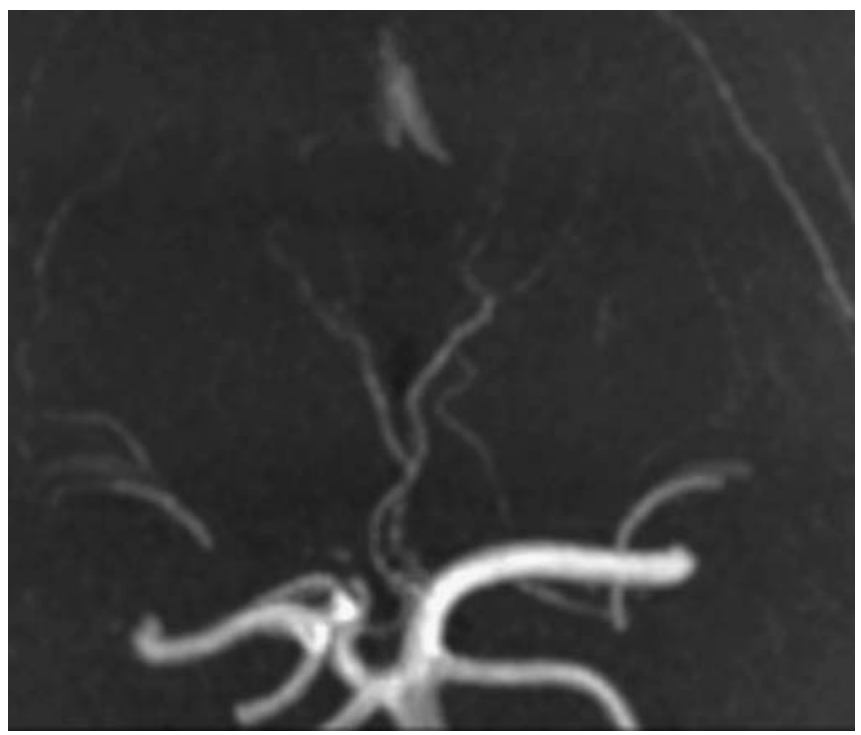

Fig. 5 Time-of-flight angiography image of the left and right P1 segment of the posterior cerebral artery (P1) with two perforating branches. One perforating artery originates from the left P1 and one originates from the right P1. Coronal slab maximum intensity projection, thickness $10 \mathrm{~mm}$ 
posterior perforating artery was associated with a larger diameter of the posterior communicating artery. Furthermore, a significant correlation was found between the diameter of the P1 and the diameter of the PCoA, and a significant correlation between the left and right halves of the posterior part of the circle of Willis for the diameter of the $\mathrm{P} 1$, the diameter of the PCoA and the P1/PCoA ratio.

In the present study with TOF MR angiography at 7.0 Tesla, we found bilateral absence of a visible PCoA in only $4.3 \%$ and unilateral absence in $15.2 \%$. In a previous study with TOF MR angiography at 1.5 Tesla, the absence of a visible PCoA was higher: the authors found bilateral absence of a PCoA in $11 \%$ of the participants and unilateral absence in $34 \%$ of the participants [12]. These results suggest that the number of unilateral or bilateral invisible PCoAs is lower at 7.0 Tesla, which shows the higher sensitivity of a smaller vasculature and a low flow at a higher field strength. According to post-mortem examinations of the circle of Willis, bilateral absence of a PCoA is very rare $(0.25 \%)$ and the unilateral absence of a PCoA is found in $2.25-3.0 \%$ [13]. Therefore, in most of the participants in the present study without a visible PCoA, this will probably be caused by low flow through the PCoA or a very small diameter. The PCoA is a unique artery that forms a connection between the anterior and posterior cerebral circulation. Thus, in the case of a balanced blood supply anteriorly and posteriorly through the circle of Willis, the blood flow through the PCoA can be very low compared with the diameter.

In our study we show the presence of a visible perforating branch originating from the $\mathrm{PCoA}$ in $64.2 \%$ of the sides on which a PCoA is visible. Compared with post-mortem studies, the current prevalence of perforators branching from the posterior communicating artery will also be an underestimation because of very low flow velocities in smaller perforating arteries, which make these arteries invisible even on the current TOF MR angiography images at 7.0 Tesla. Besides that, due to intravoxel dispersion effects ('partial volume'), signal reduction occurs. As a result the signal of these very small arteries might be too low to be detectable as an artery.

The diameter measurements of the perforating branch of the posterior communicating artery reveal that we can detect perforators with a minimum size of about $0.41 \mathrm{~mm}$. The ability to show these perforators over a length of up to approximately $36.0 \mathrm{~mm}$ in their course into the deep brain structures demonstrates the sensitivity of the current technique for these perforating arteries. Furthermore, two perforators branching from one PCoA were also detected in 2 out of 52 hemispheres.

When measuring the diameter of a vessel on an angiogram with the FWHM method, the accuracy of the FWHM method for vessels with a diameter that is close to the voxel size should be considered. The smallest artery we measured in this study was the perforating artery from the PCoA. The mean diameter of this artery was $0.72 \mathrm{~mm}$
(SD $0.15 \mathrm{~mm}$ ), so in most cases this measurement will be based on about one to two acquired pixels in the lumen of the vessel. According to a study of the accuracy of vessel diameter measurement in MR angiography, measurements with one to three pixels/diameter will give an error in the estimation of the diameter, which can be an overestimation, but even an underestimation. [14]. This error is approximately $30 \%$ at most, so in case of a vessel of $0.41 \mathrm{~mm}$, the actual diameter will lie between $0.29 \mathrm{~mm}$ and $0.53 \mathrm{~mm}$.

An overall association was found between the presence of a perforator originating from the PCoA and a larger size of the PCoA, although this was only significant for the left and right sides combined. A possible explanation for this association could be that more flow via the PCoA is needed to supply such a branch, resulting in a larger diameter of the PCoA on flow-weight MR angiography images. However, the expected amount of blood needed to feed a perforating artery is most likely too small to cause a measurable change in diameter. Therefore, we consider that the relation between a perforator branching from the PCoA has its origin in the developmental phase of the cerebral vasculature. In its embryological development there will be a higher chance of a perforator originating from a large PCoA than from a small PCoA. That the results are not significant for the left and right sides separately is possibly because of the relatively small number of participants in the study. We would expect this association to become significant when analysed in a larger study.

The correlation between the left and right halves of the posterior part of the circle of Willis, for the P1, the PCoA and the P1/PCoA ratio, shows us the relation between the blood supply of the left and the right hemispheres. If one side has a large diameter, the other side will probably also have a relatively large diameter. Not only the diameter of the P1 and the diameter of the PCoA show this relation, which can be caused by larger vessels in general within one person, but also the $\mathrm{P} 1 / \mathrm{PCoA}$ ratio is related. This indicates that the blood supplies in the left and right hemispheres, originating from the circle of Willis, are associated. As with the $\mathrm{P} 1 / \mathrm{PCoA}$ ratio itself, it is most likely that the relation between the left and right sides of the circle of Willis is also already determined in the developmental stage of the brain.

In this study we also found a significant correlation between the diameter of the $\mathrm{P} 1$ and the diameter of the PCoA, indicating that with a larger diameter of the P1, the diameter of the PCoA in general will be smaller. This could be expected because when there is more blood supply via the P1, the diameter will be larger and less blood flow via the PCoA is needed, so the diameter of the PCoA will be smaller. The observation of a smaller diameter of the PCoA suggests that the overall blood supply to the posterior part of the brain might be regulated by both $\mathrm{P} 1$ and PCoA.

The present MR angiography technique, with a detailed depiction of perforating branches of the arteries at the level of the circle of Willis, may be helpful in increasing understanding of the variations in normal anatomy in vivo 
and to improve knowledge of the pathophysiology of ischaemia in the deep brain structures. The presence of a large perforating branch from the PCoA may protect the deep brain structures, such as the thalamus, from ischaemia [2]. In addition to lacunar infarcts, the presence of perforating branches may enhance our understanding of small-vessel disease, resulting in leukoaraiosis. We speculate that the smaller amount of lacunar infarcts and whitematter lesions observed in previous studies in patients with a larger PCoA might be explained by a protective effect of the presence of a large perforating branch from the PCoA.

Although only the PCoA has been investigated in relation to ischaemic brain lesions, the collateral blood supply is also important. The anterior choroidal artery and the perforating arteries that originate from the $\mathrm{P} 1$ are important arteries to provide collateral blood supply to the area supplied by the perforating arteries originating from the PCoA. Especially in the case of ischaemic brain lesions, these vessels can play an important role in the collateral blood supply. The TOF MR angiography at 7.0 Tesla showed the bilateral presence of the anterior choroidal artery in all participants. Also the perforating branches originating from the P1 were clearly visualised in a large proportion of the $\mathrm{P} 1 \mathrm{~s}(83 \%), 63 \%$ with one visible branch and $20 \%$ with two visible branches from the P1.

In conclusion, we show the capacity of MR angiography at 7.0 Tesla to show the perforating branches of the PCoA, and by doing so, we showed the relation between the presence of these perforators and the anatomy of the PCoA. Furthermore, we found a significant correlation between the diameters of the P1 and the PCoA, and between the left and right sides of the circle of Willis. Perforating arteries originating from the $\mathrm{P} 1$ and the anterior choroidal artery are also clearly visualised with 7.0-Tesla angiography. In future studies, the detailed assessment of the perforating arteries originating from the PCoA with non-invasive MR angiography may increase our understanding of infarcts in the deep brain structures supplied by these perforating arteries.

Open Access This article is distributed under the terms of the Creative Commons Attribution Noncommercial License which permits any noncommercial use, distribution, and reproduction in any medium, provided the original author(s) and source are credited.

\section{References}

1. Schomer DF, Marks MP, Steinberg GK et al (1994) The anatomy of the posterior communicating artery as a risk factor for ischemic cerebral infarction. N Engl J Med 330:1565-1570

2. Chuang YM, Liu CY, Pan PJ, Lin CP (2008) Posterior communicating artery hypoplasia as a risk factor for acute ischemic stroke in the absence of carotid artery occlusion. J Clin Neurosci 15:1376-1381

3. Jongen JC, Franke CL, Ramos LM, Wilmink JT, van Gijn J (2004) Direction of flow in posterior communicating artery on magnetic resonance angiography in patients with occipital lobe infarcts. Stroke 35:104-108

4. van der Grond J, van Raamt AF, van der Graaf Y, Mali WP, Bisschops RH (2004) A fetal circle of Willis is associated with a decreased deep white matter lesion load. Neurology 63:14521456
5. Ikeda K, Kashihara H, Hosozawa KI et al (2005) A fetal circle of Willis is associated with a decreased deep white matter lesion load. Neurology 64:21632164

6. Fisher CM (1968) The arterial lesions underlying lacunes. Acta Neuropathol 12:1-15

7. Wardlaw JM, Dennis MS, Warlow CP, Sandercock PA (2001) Imaging appearance of the symptomatic perforating artery in patients with lacunar infarction: occlusion or other vascular pathology? Ann Neurol 50:208-215

8. Anxionnat R, Bracard S, Ducrocq X et al (2001) Intracranial aneurysms: clinical value of $3 \mathrm{D}$ digital subtraction angiography in the therapeutic decision and endovascular treatment. Radiology 218:799-808

9. Shimizu S, Suzuki H, Maki H et al (2003) A novel image fusion visualizes the angioarchitecture of the perforating arteries in the brain. AJNR Am J Neuroradiol 24:2011-2014

10. Kang HS, Han MH, Kwon BJ, Kwon OK, Kim SH, Chang KH (2005) Evaluation of the lenticulostriate arteries with rotational angiography and $3 \mathrm{D}$ reconstruction. AJNR Am J Neuroradiol 26:306-312
11. Zwanenburg JJ, Hendrikse J, Takahara T, Visser F, Luijten PR (2008) MR angiography of the cerebral perforating arteries with magnetization prepared anatomical reference at 7T: comparison with time-of-flight. J Magn Reson Imaging 28:1519-1526

12. Krabbe-Hartkamp MJ, van der Grond J, de Leeuw FE et al (1998) Circle of Willis: morphologic variation on three-dimensional time-of-flight MR angiograms. Radiology 207:103-111

13. Yasargil MG (1984) Microneurosurgery, 1st ed. Georg Thieme, Stuttgart

14. Hoogeveen RM, Bakker CJ, Viergever MA (1998) Limits to the accuracy of vessel diameter measurement in MR angiography. J Magn Reson Imaging 8:1228-1235 